\title{
EVALUACIÓN DE DOS SISTEMAS DE COMPOSTAJE CON LA ACGIÓN DE MICROORGANISMOS PARA EL MANEJO DE DESECHOS ORGÁNICOS DE LA PLANTA AGROINDUSTRIAL UTE SEDE SANTO DOMINGO
}

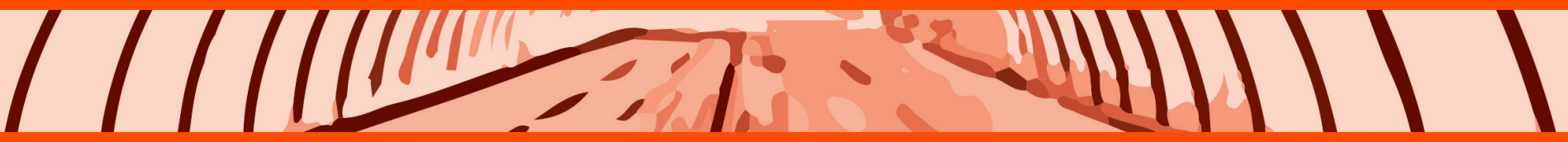

Autores:

Xavier López Mejía'

Karina Cuenca Tinoco ${ }^{2}$

Diana Quinaluisa Narváez ${ }^{3}$

${ }^{1}$ Universidad Tecnológica Equinoccial

Facultad de Ciencias de la Ingeniería, sede Santo Domingo

Email: xlopez@ute.edu.ec / Teléf.: 0997275656

${ }^{2}$ Docente Universidad Tecnológica Equinoccial

Facultad de Ciencias de la Ingeniería, sede Santo Domingo

Email: ctkc10143@ute.edu.ec / Teléf.: 0984501800

${ }^{3}$ Egresada de la Facultad de Ciencias de la Ingeniería,

Universidad Tecnológica Equinoccial, sede Santo Domingo

Recepción/Received: 2013-08-22

Email: dmqn88@hotmail.com / Teléf.: 0999629952 


\section{Resumen}

Se evaluó los sistemas de compostaje aeróbico y anaeróbico bajo la acción de microorganismos eficientes y autóctonos. Se desarrolló en la Granja Experimental de la Universidad Tecnológica Equinoccial, Escuela de Ingeniería Agropecuaria Campus Santo Domingo Arturo Ruiz Mora, ubicado km 41/2vía Santo Domingo - Chone, margen derecho en una Latitud de 000 14' S, y una Longitud de 790 11W, con el fin de identificar el mejor sistema de compostaje para desechos orgánicos de la planta agroindustrial, a más de establecer la acción de los microorganismos en cada sistema de compostaje y analizar los productos y subproductos obtenidos; determinando que el sistema aeróbico es el que en menor tiempo realizó el proceso frente al anaeróbico, la acción de los microorganismos fue similar para los autóctonos y los eficientes, los lixiviados generados poseen cantidades de nutrientes y microorganismos por lo que resulta indispensable su manejo para evitar contaminación.

Palabras Clave: Aeróbico, Anaeróbico, Compostaje, Desechos, Microorganismos, Orgánico

\section{Abstract}

In the present study, aerobic and anaerobic composting systems under the action of efficient and native microorganisms were evaluated. It was developed at the Experimental Farm of the Equinoctial Technological University, School of Agricultural Engineering Campus Arturo Ruiz Mora in Santo Domingo, located at the kilometer 4 1/2 route Santo Domingo - Chone, right margin at a latitude of 000 14' S, and a length of 790 $11^{\prime} \mathrm{W}$, in order to identify the best composting system for organic waste from the agro-industrial plant, and also to establish the action of the microorganisms in each composting system and analyze the obtained products and by-products; determining that the aerobic system performed the process in a shorter time compared to the anaerobic system, the action was similar for the native and the efficient microorganisms, the leachates generated possess quantities of nutrients and microorganisms and it is therefore essential its handling to avoid contamination.

Key Word: Aerobic, Anaerobic, Microorganisms, Organic, Waste, Composting 
Evaluación de dos sistemas de compostaje con la acción de microorganismos para el manejo de desechos orgánicos de la planta agroindustrial UTE Campus Santo Domingo

\section{Introducción}

El aumento del consumo y el avance industrial está en relación directa con el incremento de desechos producidos por el hombre, ya que en todas las actividades que él desarrolla se producen grandes cantidades (Matiz, 2011). (Tortarolo, Pereda, Palma, \& Arrigo, 2008). El compostaje es un proceso microbiológico de manejo de desechos orgánicos que en condiciones controladas, las diferentes fases térmicas que se generan dan como resultado un producto final reducido en masa y volumen, minimizando efectos adversos como olores fuertes, presencia de insectos; este producto se vuelve estable, aplicable al suelo como abono orgánico ( De Carlo, E., Rosa, A., Benintende, S., Cariello, M., Castañeda, L., Figoni, E., 2001) ( Negro, M., Villa, F., Aibar, J., Aracón, R., Ciria, P., Cristóbal, M., 2000). En la agricultura este manejo de desechos ha sido utilizado por agricultores desde hace mucho tiempo atrás existiendo referencias que en el siglo I a.C. ya manejaban estos procesos con el fin de obtener abonos para sus cosechas. (Bueno,P., Díaz,J. 2008).

En el compostaje los microorganismos actúan bajo condiciones óptimas de humedad y oxígeno que les permite consumir la materia orgánica(APROLAB, 2007). El manejo de la microbiología en el proceso de producción del compost define el éxito de la obtención del producto(Sánchez, 2009). La aplicación de microorganismos en estos procesos, mejoran la presencia de éstos en el compuesto así como el tiempo de obtención del mismo (Cariello, M., Castañeda, L., Riobo, I., González, J. 2007). La inoculación de microorganismos en el compost nos permite mantener un equilibrio en el suelo suprimiendo o controlando poblaciones patógenas por competencia y ayudando a los microorganismos nativos(APROLAB, 2007). A nivel de suelo tenemos una cantidad incalculable de microorganismos y la mayoría de éstos son oportunistas ya que imitan al grupo líder del sistema convirtiéndose en regenerativo o degenerativo (Higa, 2002). El aumento de la temperatura en este estado naciente, se basa en la actividad metabólica de un grupo de microorganismos iniciales que actúan en la materia orgánica transformándola para que un segundo grupo de microorganismos empiece su acción (De Carlo et al, 2001).
Estos cambios de temperatura en el proceso están establecidas como mesófila y termófila (Matiz, 2011). El efecto de la temperatura es de importante trascendencia en la elaboración de compost, las variaciones de ésta inciden más en la actividad microbiana que la humedad el pH o la relación Carbono/Nitrógeno ( $\mathrm{C} / \mathrm{N})$; altas temperaturas pueden afectar a los mismos microorganismos conociendo este proceso adverso como suicidio microbiano ( Bueno,P., Díaz,J., 2008).

\section{Materiales y Métodos}

\section{Ubicación}

El trabajo se realizó en la Universidad Tecnológica Equinoccial Sede Santo Domingo, en la granja experimental de la Escuela de Ingeniería Agropecuaria, ubicada km 4,5 vía Santo Domingo - Chone, margen derecho, en una Latitud de 000 14' S y una Longitud de $79011 \mathrm{~W}$

\section{Diseño experimental}

En la investigación se utilizó un Diseño Completo al Azar, con un arreglo factorial $\mathrm{A} \times \mathrm{B}$, con seis tratamientos y tres repeticiones dando un total de 18 unidades experimentales. Se evaluó la variable tiempo de descomposición de la materia orgánica, así como fluctuaciones de temperatura durante la descomposición del compostaje, realizando un análisis de varianza y una comparación múltiple de medidas por la prueba de Tukey a un nivel de significancia de 0.05 para determinar significancia estadística.

\section{Variables}

\section{Variables Independientes}

- Compostajes

- Microorganismos

\section{Variables Dependientes \\ - Temperatura \\ - Tiempo de descomposición}

\section{Tratamientos}

En el cuadro $\mathrm{N}^{\circ} 1$ se establece la descripción de los tratamientos en estudio. 
Cuadro $\mathrm{N}^{\circ}$ 1. Descripción de los tratamientos.

№ Símbolos

Tratamientos A. ME.

Aeróbico/Microorganismos Eficientes

2 A. MA

Aeróbico/ Microorganismos Autóctonos

$3 \quad$ AN.ME.

Anaeróbico/Microorganismos Eficientes

$4 \quad$ AN.MA.

Anaeróbico/ Microorganismos Autóctonos

$5 \quad$ A.T

6 AN.T.

Aeróbico/ Testigo

Anaeróbico/ Testigo

\section{Característica del área experimental}

En el cuadro $\mathrm{N}^{\circ} 2$ se describe el área experimental

Cuadro $\mathrm{N}^{\circ}$ 2. Descripción del Área

\begin{tabular}{|c|c|}
\hline Descripción & Cantidad \\
\hline Área total del Experimento: & $224 m^{2}$ \\
\hline Área útil para el experimento: & $100 m^{2}$ \\
\hline Kg total de Materia Orgánica: & $1062 \mathrm{~kg}$ \\
\hline Kg por tratamiento de Materia Orgánica: & $59 \mathrm{~kg}$ \\
\hline Numero de U. Experimentales: & 18 Unidades \\
\hline
\end{tabular}

\section{Datos tomados y métodos de evaluación}

\section{Toma de temperatura}

La toma de temperatura se realizó dos veces por semana, a las $16 \mathrm{Hoo}$, estableciendo tres datos por tratamiento.

\section{Tiempo de descomposición de los desechos orgánicos}

La medida utilizada para determinar el tiempo de descomposición fue en días, se tomó en cuenta desde el día de la instalación hasta el día de la cosecha.

\section{Manejo del experimento}

\section{Establecimiento de los tratamiento}

Se construyó plataformas de tierra con una pequeña pendiente para drenar los lixiviados en los compuestos aeróbicos; para las composteras anaeróbicas que se encontraban en recipientes cubiertos, se realizó un orificio en la base de los mismos para recolectar los lixiviados producidos.

Para los dos sistemas se colocó el desecho orgánico previamente picado y mediante un sorteo al azar se distribuyó cada uno de los tratamientos en estudio, en los cuales se depositó $59 \mathrm{Kg}$. de materia orgánica preparada

\section{Aplicación de los microorganismos}

Para la activación de microorganismos se colocó 1litro de solución madre del compuesto, 2 litros de melaza y 17 litros de agua dejando reposar por 15 días para llegar a un $\mathrm{pH}$ de 3,5. De esta solución preparada se aplicó 3 litros de microorganismos activados correspondientes a cada tratamiento.

\section{Cosecha}

Se realizó cuando cada uno de los diferentes tratamientos presentaron un color café oscuro, con olor a mantillo de bosque y una temperatura constante.

\section{Resultados}

Análisis del comportamiento de la temperatura vs. el tiempo de compostaje para los tratamientos aerobios con respecto al testigo

En la primera semana la temperatura favoreció condiciones adecuadas para el desarrollo de microorganismos mesófilos, en el caso del testigo, registrándose temperaturas iniciales correspondientes a la etapa mesotérmica $\left(10-40^{\circ} \mathrm{C}\right)$, la cual perduró entre la primera y doceava semana; en los tratamientos eficientes y autóctonos el desarrollo de mesófilos se hizo presente en el segundo y tercer día; al cuarto día los microorganismos termófilos hacen su 
Evaluación de dos sistemas de compostaje con la acción de microorganismos para el manejo de desechos orgánicos de la planta agroindustrial UTE Campus Santo Domingo

presencia, luego de ello empieza la etapa de enfriamiento; en la sexta semana hay un repunte en la temperatura, este comportamiento se originó probablemente por la temperatura ambiental que influyó en el comportamiento del mismo. La etapa termogénica $\left(40-75^{\circ} \mathrm{C}\right)$ tuvo una duración de 4 días. La segunda etapa mesotérmica o de enfriamiento $\left(\leq 40^{\circ} \mathrm{C}\right)$ tuvo una duración de 2 semanas, llegando a registrarse temperaturas entre 31 y $39^{\circ} \mathrm{C}$. Estos valores son superiores a la temperatura ambiente por lo que se estima que el proceso de compostaje sigue en la etapa activa y que aún requiere de un periodo de descomposición, antes de dar lugar a la fase de maduración hasta alcanzar valores estables y cercanos a la temperatura ambiente.

Las experiencias consultadas registran tiempos de maduración en promedio de 40-50 días para la obtención de un compost estable a una temperatura final de $25^{\circ} \mathrm{C}$. Por lo que como se puede apreciar en el gráfico $\mathrm{N}^{\circ} 1$ la fase de maduración (en la que se puede apreciar el olor y color característico del compost) está presente desde la semana novena y doceava para los tres casos de tratamientos. En los resultados obtenidos tanto la utilización de microorganismos eficientes como los microorganismos autóctonos tienen similar comportamiento y se ve influenciado los resultados en comparación con el tratamiento testigo.

Grafico $\mathrm{N}^{\circ} 1$. Comportamiento de la temperatura vs el tiempo de compostaje para los tratamientos aerobios con respecto al testigo

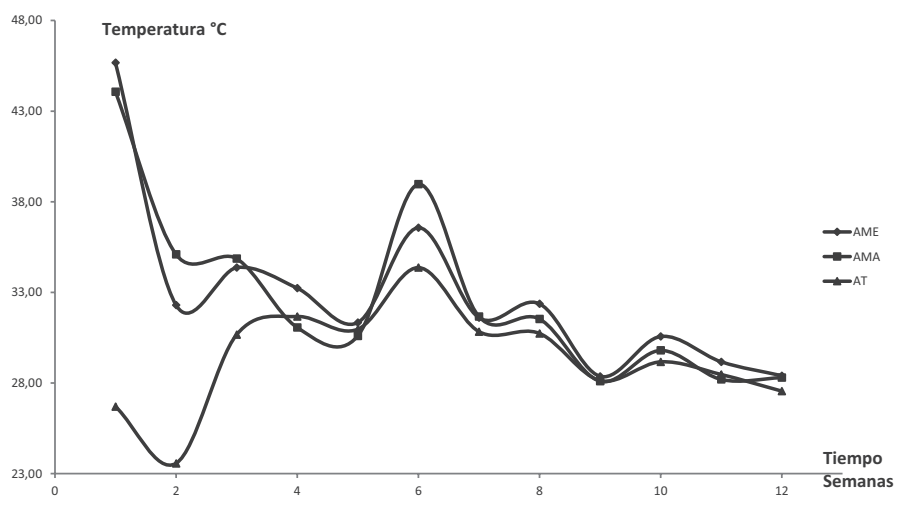

Análisis del comportamiento de la temperatura vs. el tiempo de compostaje para los tratamientos anaeróbicos con respecto al testigo

Como se puede apreciar en el gráfico $\mathrm{N}^{\circ} 2$ los valores de temperatura registrados indican que el proceso no llegó a la etapa termogénica, esto se hizo notorio puesto que no se eliminaron los microorganismos mesófilos patógenos como hongos y esporas. A estas condiciones tanto el testigo como los tratamientos con microorganismos eficientes y autóctonos tienen similar comportamiento, existiendo repuntes de temperatura con el pasar de las semanas que no difieren del tipo de tratamiento. Estos microorganismos no pueden destruir con facilidad las moléculas celulósicas del compost por lo que se demoran más tiempo en desdoblar dicha materia orgánica, requiriendo en este caso entre quince y dieciséis semanas para la obtención del compost.

Grafico $\mathrm{N}^{\circ}$ 2. Comportamiento de la temperatura vs el tiempo de compostaje para los tratamientos anaerobios con respecto al testigo

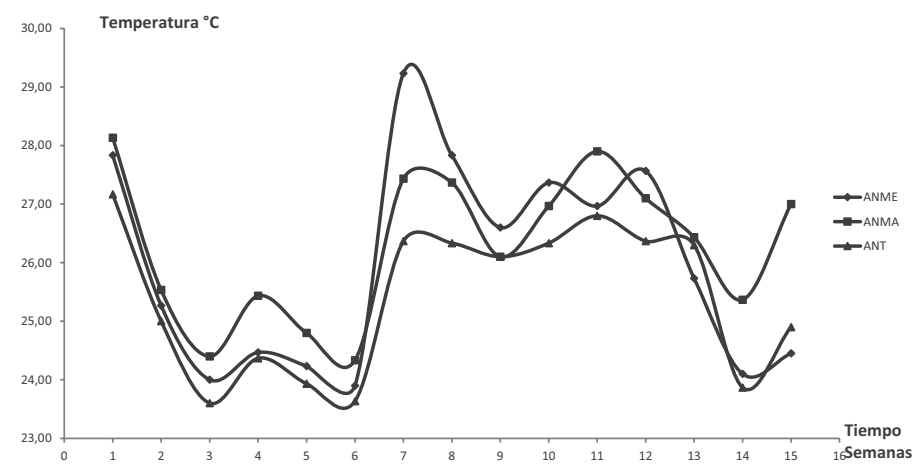

\section{Generación de lixiviados}

Durante el proceso de formación de compost, se generó lixiviado en todos los tratamientos, estos fueron recogidos en material plástico para no contaminar en suelo.

indica que el control de generación de lixiviado es un factor importante debido a los posibles contaminantes que generan durante el compostaje de residuos agrícolas como el nitrógeno en forma de nitratos (NO3-). Las pilas almacenadas y en proceso de maduración son fuentes potenciales de nitratos La lixiviación de nitrógeno a través del suelo puede contaminar las fuentes de suministro de agua, esto implica problemas sanitarios si el agua es usada para el consumo humano (Yañez,P., Levy,A., Azero, M. 2007), debido a ello se mantuvo un control primordial en la recolección del lixiviado, mismo que fue analizado como se puede apreciar en los cuadros 2 y 3. 
Cuadro $\mathrm{N}^{\circ}$ 2. Composición de macronutrientes en \% de materia seca del lixiviado

\begin{tabular}{lcccccc}
\hline \multirow{2}{*}{ Tratamientos } & \multicolumn{5}{c}{ Macro nutrientes en \% de materia seca } \\
\cline { 2 - 7 } & $\mathbf{N}$ & $\mathbf{P}$ & $\mathbf{K}$ & $\mathbf{C a}$ & $\mathbf{M g}$ & $\mathbf{S}$ \\
A ME & 0,44 & 0,05 & 4,17 & 2,92 & 0,30 & 0,52 \\
A MA & 0,36 & 0,04 & 3,95 & 3,09 & 0,33 & 0,50 \\
AN ME & 0,14 & 0,04 & 0,72 & 2,29 & 0,07 & 0,09 \\
AN MA & 0,17 & 0,05 & 0,81 & 2,48 & 0,09 & 0,14 \\
A T & 0,25 & 0,05 & 4,06 & 2,70 & 0,33 & 0,49 \\
AN T & 0,14 & 0,05 & 0,60 & 2,46 & 0,09 & 0,06 \\
\hline
\end{tabular}

Cuadro $\mathrm{N}^{\circ}$ 3. Resultado de la composición en microorganismos para los lixiviados obtenidos

\begin{tabular}{ccc}
\hline Tratamientos & Mohos y Levaduras & Bacterias \\
\hline A ME & 150000 & 20000000 \\
A MA & 100000 & 40000000 \\
AN ME & 10 & 2100000 \\
AN MA & 5000 & 6000000 \\
A T & 18000 & 800 \\
AN T & 40000 & 82 \\
\hline
\end{tabular}

\section{Calidad de Compost obtenido}

El abono orgánico obtenido luego del proceso de compostaje en este trabajo, obtuvo como indicadores característicos un color oscuro y un olor a tierra de montaña no intervenida, estas tipologías garantizan una buena presencia de nutrientes y vida microbiológica aumentando la solubilización de los nutrientes haciendo que éstos sean mejor asimilados por las raíces además de mejorar las características físicas, químicas y biológicas de los suelos. Los resultados del análisis químico para macro y micronutrientes se presentan en los cuadros 4 y 5 respectivamente, el análisis físico químico en el cuadro 6 y la composición micro organismos en el cuadro 7.

Cuadro $\mathrm{N}^{\circ}$ 4. Resultado de la composición de macronutrientes en \% de materia seca del compost obtenido por cada tratamiento

\begin{tabular}{ccccccc}
\hline \multirow{2}{*}{ Tratamientos } & \multicolumn{7}{c}{ Macro nutrientes } \\
\cline { 2 - 7 } & $\mathbf{N}$ & $\mathbf{K}$ & $\mathbf{C a}$ & $\mathbf{M g}$ & $\mathbf{s}$ & $\mathbf{P}$ \\
AME & 2,00 & 4,46 & 3,35 & 0,49 & 0,22 & 0,38 \\
AMA & 1,50 & 4,00 & 3,24 & 0,57 & 0,26 & 0,39 \\
ANME & 3,90 & 0,24 & 3,78 & 0,37 & 0,24 & 0,31 \\
ANMA & 2,50 & 2,20 & 3,86 & 0,43 & 0,16 & 0,39 \\
AT & 2,20 & 2,72 & 3,78 & 0,47 & 0,21 & 0,36 \\
ANT & 3,50 & 1,91 & 3,45 & 0,31 & 0,15 & 0,33 \\
\hline
\end{tabular}

Cuadro $\mathrm{N}^{\circ}$ 5. Resultado de la composición de micronutrientes en partes por millón para el compost obtenido por cada tratamiento

\begin{tabular}{cccccc}
\hline & \multicolumn{5}{c}{ Micronutrientes } \\
Tratamientos & Cu & Zn & Mn & B & Fe \\
\cline { 2 - 6 } AME & 35 & 79 & 108 & 36,85 & 606 \\
AMA & 35 & 75 & 69 & 57,77 & 56,5 \\
ANME & 28 & 70 & 131 & 34,91 & 528 \\
ANMA & 34 & 76 & 143 & 27,93 & 550 \\
AT & 31 & 69 & 108 & 38,35 & 604 \\
ANT & 33 & 74 & 152 & 29,9 & 562
\end{tabular}

Cuadro $\mathrm{N}^{\circ}$ 6. Resultado de los análisis físico - químico al compost obtenido en todos los tratamientos

\begin{tabular}{ccccc}
\hline & \multicolumn{4}{c}{ Análisis físico químico } \\
Tratamiento & \multicolumn{4}{c}{ Humedad } \\
\cline { 2 - 5 } & $\mathbf{p H}$ & C. E ds/m & M .0 \% & $\%$ \\
AME & 8,52 & 62,92 & 31,27 & 71,02 \\
AMA & 9,30 & 0,77 & 43,91 & 71,02 \\
AT & 8,46 & 11,79 & 33.93 & 63,31 \\
ANME & 6,87 & 4,25 & 26,44 & 77.36 \\
ANMA & 8,72 & 3,80 & 21,47 & 73,31 \\
ANT & 8,37 & 2,57 & 22,09 & 72,08 \\
\hline
\end{tabular}

Cuadro $\mathrm{N}^{\circ}$ 7. Resultado de la composición en microorganismos para todos los compost obtenidos

\begin{tabular}{crc}
\hline Tratamientos & $\begin{array}{c}\text { BACTERIAS } \\
\text { ufc/ml }\end{array}$ & $\begin{array}{c}\text { MOHOS Y LEVADURAS } \\
\text { upc/ml }\end{array}$ \\
\cline { 2 - 3 } AME & 16000000 & 1400000 \\
AMA & 60000000 & 5200000 \\
AT & 2500000 & 32000 \\
ANME & 120000000 & 400000 \\
ANMA & 70000000 & 50000 \\
ANT & 200000 & 16000 \\
\hline
\end{tabular}

Análisis de los resultados obtenidos del compost

De acuerdo a los análisis realizados para determinar las características químicas y biológicas a los compost obtenidos en cada 
Evaluación de dos sistemas de compostaje con la acción de microorganismos para el manejo de desechos orgánicos de la planta agroindustrial UTE Campus Santo Domingo

tratamiento se establece que están en relación a la calidad y cantidad de material utilizado y al tipo de microorganismos manejado en el proceso obteniendo un producto con color obscuro, sin mal olor, con homogeneidad en los contenidos de macro y micronutrientes, existiendo diferencias en el conteo microbiológico por el mismo hecho de utilizar diferentes tipos de microorganismos para el estudio

\section{Conclusiones}

Con base en los resultados obtenidos en la investigación se logró llegar a las siguientes conclusiones:

Durante el proceso de descomposición de los desechos orgánicos de la planta agroindustrial, los tratamientos que se realizaron con el tipo de compostaje Aeróbico, ( A.ME., A.MA. y A.T.), obtuvieron mayor temperatura. Presentando el T1 (aeróbico con $M$. Eficientes) una temperatura de $32,93^{\circ} \mathrm{C}$.

Los compostajes tipo Aeróbico, (A.ME., A.MA. y A.T.), estuvieron listos para la cosecha a los 72 días, logrando el menor tiempo en la descomposición de los desechos orgánicos.

En los lixiviados y en el compostaje los tratamientos de tipo aeróbico con microorganismos (Autóctonos, Eficientes) presentaron mayor cantidad de bacterias, mohos y levaduras.

Presentan mayor cantidad de nutrientes los lixiviados y compostaje que corresponden a los tratamientos de tipo aeróbico con microorganismos (Autóctonos, Eficientes).

El mejor tipo de compostaje para el manejo de los desechos orgánicos de la planta agroindustrial es el tipo aeróbico ya que presentó menos tiempo para la obtención del mismo.

\section{Referencias Bibliográficas}

APROLAB. (2007). Manual para la producción de compost con microorganismos eficaces. 22.

Bueno, P., \& Díaz, J. (2008). Factores que afectan al proceso de Compostaje. In J. Moreno \& R. Moral (Eds.), Compostajes (pp. 93 - 110). Madrid

Cariello, M., Castañeda, L., Riobo, I., \& González, J. (2007). Endogenous microorganisms inoculant to speed up the composting process of urban swage sludge. Revista de la ciencia del suelo y nutrición vegetal, 7, 26-35.

De Carlo, E., Rosa, A., Benintende, S., Cariello, M., Castañeda, L., Figoni, E., et al. (2001). Estudio de la población microbiana en las etapas iniciales del compostage. Revista Ceres, XLVIII, 17.

Higa, T. (2002). Una revolución para salvar la tierra. Okinawa.

Matiz, A. (2011). Aprovechamiento de residuos sólidos orgáncos mediante procesos microbiológicos en puro Indira - Guainía. 1-7.

Negro, M., Villa, F., Aibar, J., Aracón, R., Ciria, P., Cristóbal, M., et al. (2000). Producción y gestión del compost. 31.

Sanchéz, T. (2009). Caracterización microbiológica del proceso de compostaje a partir de residuos azucareros. Agronomía Tropical, 59.

Tortarolo, M., Pereda, M., Palma, M., \& Arrigo, N. (2008). Influencia de la inoculación de microorganismos sobre la temperatura en el proceso de compostaje. Ciencia del suelo, 26, 41-51.

Yañez, P., Levy, A., \& Azero, M. (2007). Evaluación del compostaje de residuos de dos agroindustrias palmiteras del Trópico de Cochabamba en silos hiperventilados. [Artículos Científicos]. 3, 16 . 
Anexos

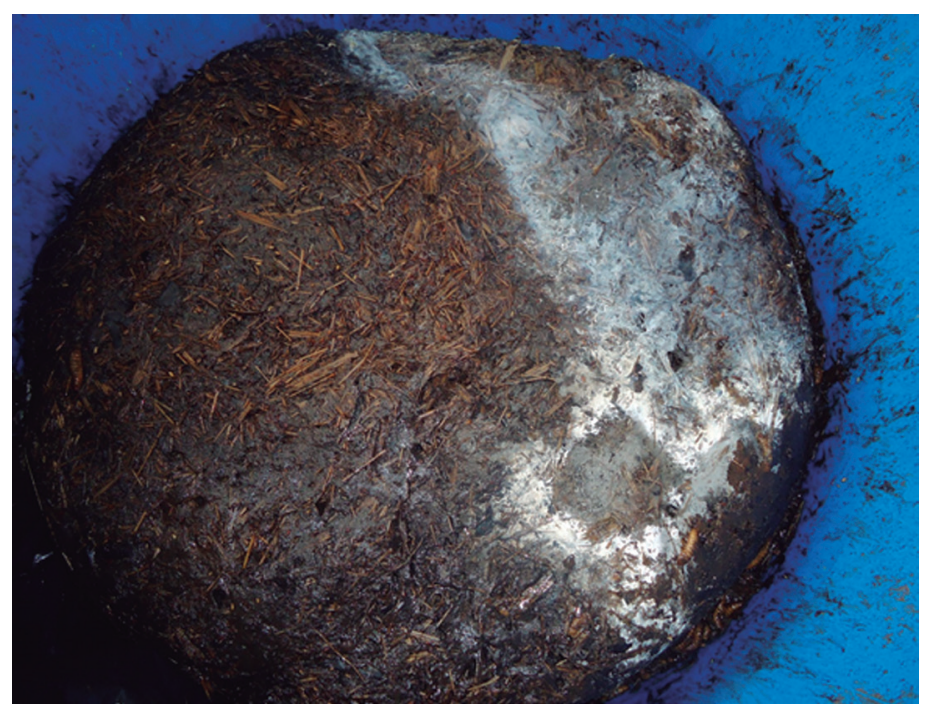

Microorganismos Colonizando el Sistema de Compostaje Anaeróbico

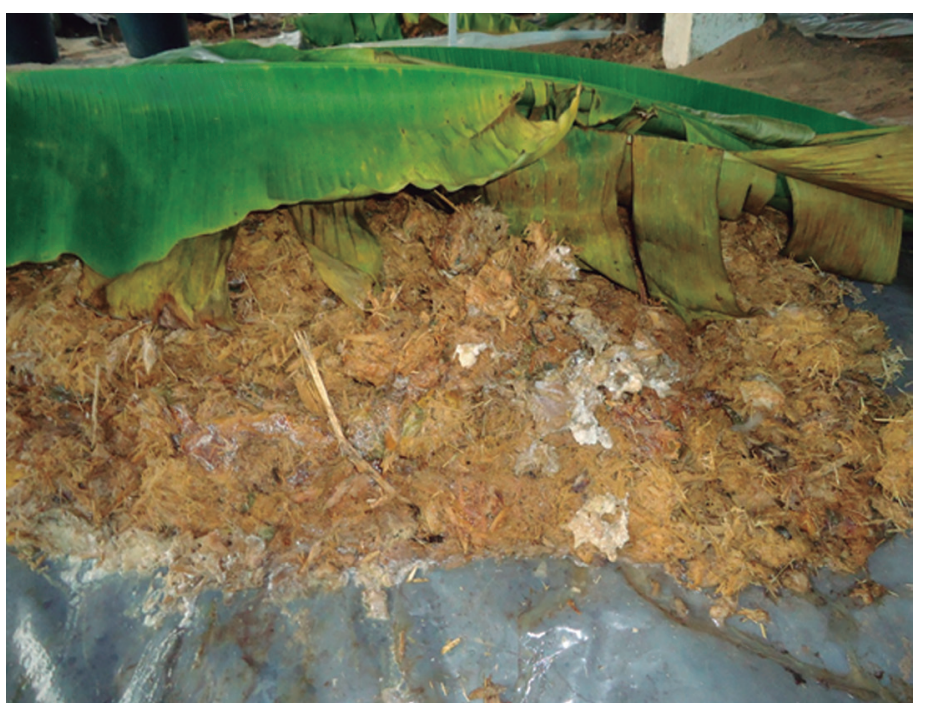

Colonias de Microorganismos en el Compost Aeróbico 